\title{
rAAV vector-mediated gene therapy for experimental ischemic stroke
}

\author{
Zhao-Jian Li, Ren-zhi Wang \\ Department of Neurosurgery, Peking Union Medical College Hospital, Peking Union Medical College, Beijing, 100730, China
}

\begin{abstract}
The safest viral vector system for gene therapy is based on recombinant adeno-associated virus (rAAV) up to date in Phase I clinical trials, which has been developed rapidly and applied for ischemic stroke gene therapy in animal experiments since the past seven years. rAAV vector has made great progress in improving gene delivery by modification of the capsid and increasing transgene expression by encapsidation of double-stranded rAAV genome. And in all, nine therapeutic genes in 12 animal studies were successfully delivered using rAAV vector to ischemic brain via different approaches in rat or mice stroke models for gene therapy and the results suggested that rAAV could mediate genes' expression efficiently; most of them displayed evidently therapeutic efficacy with satisfactory biological safety. Gene therapy involving rAAV vector seems effective in attenuation of ischemic damage in stroke and has greatly promising potential use for patients in the future. In this review, we will focus on the basic biology and development of rAAV vector itself as well as the recent progress in the use of this vector for ischemic stroke gene therapy in animal experiments.
\end{abstract}

Key words: Gene therapy, ischemic stroke, recombinant adeno-associated virus, vector

Stroke is the leading cause of disability and the third leading cause of death in major industrialized countries and about $85 \%$ strokes are ischemic. ${ }^{[1]}$ However, an effective treatment for this condition has not been established apart from the thrombolytic recombinant tissue plasminogen activator, which must be given within $3 \mathrm{~h}$ of stroke onset. ${ }^{[1]}$ Gene transfer is an attractive method for the treatment of many central nervous system (CNS) disorders and ischemic stroke is a target of gene therapy. ${ }^{[2]}$

Several viral systems, including retrovirus, recombinant herpes simplex virus, adenovirus and more recently, recombinant adeno-associated virus (rAAV) and Sendai virus, have been used as vectors for gene delivery to ischemic stroke. Of these vectors, adenovirus is the most commonly used, but there are substantial limitations associated with the clinical application of this vector for its short-term expression, adverse inflammatory and immunologic responses ${ }^{[3]}$ As a useful gene therapy alternative vehicle to adenovirus, rAAV vectors exhibit a number of attractive properties for both gene therapy for CNS diseases and basic neurobiological investigations. The past seven years have witnessed some advancement in rAAV-mediated gene therapy for ischemic stroke and in this article we will attempt to focus on rAAV-based research for ischemic stroke gene therapy.

\section{Characteristics and Progress of rAAV Vector}

Adeno-associated virus (AAV) is a nonenveloped virus of the parvovirus family with a $4.7 \mathrm{~kb}$, linear singlestranded DNA genome. To date, there are approximately 35 known serotypes of AAV and over 100 variants have been isolated from human or nonhuman primate tissues and at least 10 serotypes have been engineered into rAAV vectors. ${ }^{[4]}$ After the establishment of the first infectious clone of AAV2 in 1982, rAAV2 vector rapidly gained popularity in gene therapy applications for its safety. Until recently, the majority of the research conducted using AAV-based vectors employed serotype rAAV2. In recent years, with increased enthusiasm, more and more studies in rAAV vectors have paid much attention to new serotype vector, mixed capsid vector, double-stranded vector and tropism-modified vector and it was reported that rAAV8, rAAV1 and rAAV5 have higher transduced distribution and number of neurons than rAAV2 in the CNS. ${ }^{[5,6]}$

The AAV transfection involves a multistep process, which begins with virus binding to the cell surface and followed by viral uptake, intracellular trafficking, nuclear localization, uncoating and the synthesis of second-strand DNA. ${ }^{[7-12]}$ The AAV2 initiates infection by binding to its primary receptor, heparan sulfate proteoglycans (HSPG). ${ }^{[7]}$ In addition, as two coreceptors for AAV2 infection, ${ }^{[8-10]}$ fibroblast growth factor 
receptor 1 (FGFR1) probably functions to enhance the viral attachment process, ${ }^{[9,10]}$ while $\alpha_{\mathrm{v}} \beta_{5}$ integrin may involve viral endocytosis..$^{[10,12,13]}$ Furthermore, AAV2 binding to the cell surface via $\alpha_{v} \beta_{5}$ integrin may activate Rac1, which induces the stimulation of phosphoinositol-3 kinase, culminating in the rearrangements of the microfilaments and microtubules that support trafficking of AAV2 to the nucleus after endocytosis. ${ }^{[13]}$

The advantages of rAAV vector for ischemic stroke gene therapy include: (1) their nonpathogenicity and low immunogenicity; (2) broad host and cell type tropism range; (3) the ability to transduce both dividing and nondividing cells; (4) the possible integration of the gene into the host genome; (5) easy production in high titer; (6) the long-term stable expression of transgenes. ${ }^{[3]}$ Infection by the wild-type AAV is not associated with any human disease, even though serological evidence suggested that $60-80 \%$ of adults had been exposed to AAV. ${ }^{[14]}$ It is the ability of AAV to become latent without self-replication that originally made this virus very attractive as a gene transfer tool. In addition, the tropism of rAAV vector can be modified to transduce specific tissue or cell through adding a bridge molecule, direct modification of the capsid protein or the application of different promoters. Although the existence of blood-brain barrier (BBB) has greatly reduced the efficacy of viral vectors-mediated gene therapy for CNS disorders, rAAV vector may cross disrupted BBB following ischemia more easily and efficiently compared to adenovirus vector, for its particle diameter is only $20 \mathrm{~nm} \cdot{ }^{[14]}$ And it is also possible that both proliferating endogenous neural stem cells and injured matured cells following ischemia may become rAAV transferred targets now that it can transduce both dividing and nondividing cells. ${ }^{[15]}$ With above great potential superiorities, rAAV vectors may display promising perspective for ischemic stroke gene therapy in the future.

Initially, the administration of rAAV vector in gene therapy was limited to its low titer, which was insufficient to transfect cells or tissue efficiently in vivo or in vitro. With improvement in its production technique, new production protocols allow for high titer and can be scaled up. ${ }^{[16]}$ Limited packaging capacity is the other clear limitation of rAAV as vector system and the transgene and promoter cassette must be 4.7-5.0 kb, which typically will not allow rAAV to be used for larger cDNAs. However, most therapeutic genes for stroke treatment fall into the above range and it is also possible, via mutagenesis studies, to create an active minigene as has been done successfully with the gene dystrophin, which was mutated in muscular dystrophy. ${ }^{[17]}$ In addition, delayed expression of rAAV-mediated transgene, due to the requirement of conversion of its single-stranded DNA to double-stranded DNA, may be another disadvantage for acute ischemic stroke gene therapy, in which therapeutic gene needs to be expressed as early as possible to rescue or protect ischemic cells. Fortunately, the recent development of double-stranded rAAV vectors has eliminated the need for second strand synthesis, leading to much earlier and higher transduction efficiencies in vitro and in vivo; ${ }^{[18]}$ so double-stranded rAAV will be more effective and promising for stroke gene therapy than single-stranded rAAV. Moreover, for some therapeutic factors, long-lasting gene expression mediated by rAAV vector in ischemic or normal brain tissue may cause side-effects, especially in the recovery phase of stroke. For resolving this problem, Shen et al., ${ }^{[19]}$ recently reported that introduction of a hypoxia-responsive promoter in rAAV vector construction was very helpful for driving therapeutic gene-specific expression in brain tissue with hypoxia and its expression attenuated with amelioration of hypoxia, which reduced the detrimental effect of vascular endothelial growth factor (VEGF) in mice stroke model.

\section{rAAV-Mediated Gene Therapy in Animal Stroke Model}

The pathophysiology of ischemic stroke is complex and incompletely understood until now. Of the numerous pathways identified, excessive activation of glutamate receptors, accumulation of intracellular calcium cations, abnormal recruitment of inflammatory cells, excessive production of free radicals and initiation of pathological apoptosis are believed to play critical roles in ischemic damage, especially in the penumbra. For the last decade, gene therapy with rAAV vectors has displayed evident curative effect in animal stroke models by increasing glucose uptake and neovascularization, inhibiting apoptosis, inflammation, excitotoxicity and free radicals, reducing cytosolic calcium and protecting proliferating endogenous precursor cells. In these studies, two main types of rodent animal stroke models, the transient middle cerebral artery occlusion (tMCAO) model and transient global cerebral ischemia (tGCI) model, were involved. And to date, only nine therapeutic genes in 12 experiments have been delivered using rAAV vectors into the ischemic hemisphere for gene therapy via different vector delivery approaches [Table 1].

\section{Growth factor genes}

More than 30 growth factors have been identified and many of them are reported to have neuroprotective effects for brain ischemia injury. ${ }^{[20]}$ However, only a few genes of these neurotrophins, including brain-derived neurotrophic factor (BDNF), glial cell line-derived neurotrophic factor (GDNF) and nerve growth factor (NGF), have been delivered via rAAV vector for stroke gene intervention. Moreover, rAAV-BDNF and rAAV- 


\begin{tabular}{|c|c|c|c|c|c|c|}
\hline \multirow{4}{*}{$\begin{array}{l}\text { Transgene } \\
\text { BDNF }\end{array}$} & Stroke model & rAAV serotype & Delivery approach & Delivery time & Authors & Yon \\
\hline & tMCAO & rAAV2 & striatum & $4-5$ w before ischemia & Andsberg et al. & $2002^{[26]}$ \\
\hline & tMCAO & rAAV2 & substantia nigra & $4-5$ w before ischemia & Gustafsson et al. & $2003^{[27]}$ \\
\hline & unknown & rAAV2 & hippocampus & unknown & Larsson et al. & $2002^{[28]}$ \\
\hline \multirow[t]{2}{*}{ GDNF } & tMCAO & rAAV2 & substantia nigra, striatum & before ischemia & Arvidsson et al. & $2003^{[34]}$ \\
\hline & tMCAO & rAAV2 & cortex & during ischemia & Tsai et al. & $2000^{[33]}$ \\
\hline \multirow[t]{2}{*}{$\mathrm{Bcl}-2$} & $\mathrm{tGCl}$ & rAAV2 & hippocampus & $\begin{array}{l}5 \mathrm{~d} \text { before ischemia, } \\
1 \mathrm{~h} \text { after ischemia }\end{array}$ & Shimazaki et al. & $2000^{[35]}$ \\
\hline & $\mathrm{tGCl}$ & rAAV2 & hippocampus & after ischemia & Okada et al. & $2002^{[36]}$ \\
\hline BCL-w & tMCAO & rAAV2 & cortex, striatum & $3 \mathrm{w}$ before ischemia & Sun et al. & $2003^{[37]}$ \\
\hline \multirow[t]{2}{*}{ VEGF } & $\mathrm{tGCl}$ & rAAV2 & lateral ventricle & $6 \mathrm{~d}, 12 \mathrm{~d}$ before ischemia & Bellomo et al. & $2003^{[40]}$ \\
\hline & tMCAO & rAAV2 & lateral ventricle & $5 \mathrm{~d}$ before ischemia & Shen et al. & $2006^{[19]}$ \\
\hline NGF & tMCAO & rAAV2 & striatum & $4-5$ w before ischemia & Andsberg et al. & $2002^{[26]}$ \\
\hline IL-1ra & $\mathrm{tGCl}$ & rAAV2 & cortex & after ischemia & Tsai et al. & $2003^{[44]}$ \\
\hline Neuroglobin & tMCAO & rAAV2 & cortex, striatum & $3 w$ before ischemia & Sun et al. & $2003^{[45]}$ \\
\hline AIP & $\mathrm{tGCl}$ & rAAV2 & hippocampus & $5 \mathrm{~d}$ before ischemia & Cao et al. & $2004^{[46]}$ \\
\hline
\end{tabular}

GDNF attract more attention than rAAV-NGF for their inconsistent efficacy in stroke experimental treatment.

Brain-derived neurotrophic factor is a neurotrophin that promotes the survival and growth of developing neurons in vitro ${ }^{[21,22]}$ and improves motor neuronal functions in animal models. ${ }^{[23,24]} \mathrm{In}$ animals subjected to transient forebrain ischemia, BDNF was neuroprotective and attenuated ischemic neuronal injury. ${ }^{[25]}$ Delayed treatments with the BDNF chimeric peptide showed an effective time window of one to two hours after ischemia. ${ }^{[20]}$ In the experiment by Andsberg et al., ${ }^{[26]}$ rAAV2-BDNF was infused into the striatum four to five weeks before ischemia in a rat $\mathrm{MCAO}$ model and there was only a mild functional benefit in the treatment group, which was concomitant with a mild protective effect on peptidergic striatal interneurons. On the contrary, in another study exploring the hypothesis that paracrine BDNF delivery might be more efficacious in the tMCAO model than autocrine BDNF release, rAAV2BDNF injection into the substantia nigra, which induced high levels of BDNF release in the ipsilateral striatum via retrograde axonal transport, resulted in more cells' death than that seen in rAAV2-GFP-treated controls. ${ }^{[27]}$ Similarly, it was also shown that rAAV2-mediated hippocampal BDNF delivery suppressed ischemiainduced neurogenesis in rat stroke model. ${ }^{[28]}$

Several findings suggest that GDNF may affect striatal neurogenesis after stroke, ${ }^{[29]}$ which acts via the extracellular glycosylphospatidylinositol-linked receptor, GDNF family co-receptor $\alpha 1$ and the transmembrane tyrosine kinase, c-Ret or through a c-Ret-independent mechanism. ${ }^{[30]}$ It was also reported that GDNF receptor expression was upregulated in the penumbral areas following cerebral ischemia in rats $^{[31]}$ and short-term neuronal survival is improved by GDNF delivery. ${ }^{[32]}$ Recently, cortical injection of rAAV2-GDNF immediately after tMCAO has been reported to protect cortical cells by the mechanism of preventing apoptosis and showed that rAAV2 was a potential delivery vector of GDNF gene for the therapy of stroke. ${ }^{[33]}$ In contrast, long-term, rAAV-mediated high levels of GDNF can exacerbate neuronal loss and the result from Arvidsson et al., ${ }^{[34]}$ found that high level expression of rAAV2-GDNF in striatum before ischemia provided no protection to striatal neurons at five or eight weeks after cerebral ischemia and moreover, supply of rAAV2-GDNF did not alleviate the striatumrelated behavioral deficits. In addition, nerve growth factor (NGF), as the name implies, is an endogenous neurotrophic factor for central cholinergic neurons. In an animal study involving a rat tMCAO model Andsberg et al., ${ }^{[26]}$ showed that intrastriatal delivery of rAAV2-NGF could moderately mitigate neuronal death following stroke, which led to detectable functional sparing in model rats.

Thus, the above data may indicate that the actions of rAAV-BDNF and rAAV-GDNF in ischemic stroke are more complex than previously believed and more studies are necessary to further validate their efficacy for stroke or to investigate whether rAAV-meidated delayed expressions of therapeutic genes influence their protective effects for acute cerebral ischemia. For slow onset of rAAV-mediated therapeutic gene expression, which is believed to be related to conversion of singlestranded rAAV vector genome to double-stranded templates, gene therapy before ischemia may show better efficacy than after ischemia, now that vector administration in the former has longer time to express higher level of growth factor for early protection against ischemic injury in stroke.

\section{Anti-apoptosis genes}

Inhibition of apoptosis mediators may be one of the gene therapeutic principles for stroke. Bcl-2 and BCL-w are known as two anti-apoptotic genes that confer the ability to block neuronal cell programmed death after brain ischemia. For examining the protective effect of Bcl-2 on delayed neuronal death in the CA1 region of gerbil hippocampus after transient ischemia, Shimazaki et al., ${ }^{[35]}$ injected rAAV-bcl-2 into the CA1 pyramidal cell 
layer and found that the application of rAAV2-bcl-2 both before ischemia and after ischemia could prevent DNA fragmentation in CA1 neurons. Likewise, in the study of bcl-2 gene transfer to gerbil hippocampus for the treatment of reperfusion injury, Okada et al., ${ }^{[36]}$ showed that postischemic injection of rAAV vector was effective for bcl-2 gene delivery conferring neuroprotection in the gerbil hippocampus. Similarly, by local intracerebral administration of rAAV2-BCL-w to rat cerebral cortex and striatum three weeks before focal cerebral ischemia induced by tMCAO, Sun et al., ${ }^{[37]}$ found BCL-w expression was increased in cerebral cortex and striatum neurons, astroglia and endothelial cells and recipients of the rAAV-BCL-w also showed a $30 \%$ reduction in infarct size and a $33-40 \%$ improvement in neurological function, compared to the control groups. These results implied that rAAV-based anti-apoptosis gene intervention prior to ischemia may be a rational and effective therapeutic strategy for stroke.

\section{Angiogenesis genes}

Many studies have shown that neovascularization induced by growth factors may represent a rational therapy for patients with stroke. Vascular endothelial growth factor (VEGF) plays a pivotal role in therapeutic angiogenesis and VEGF expression is enhanced in the post-ischemic brain. And a lot of animal studies have suggested that VEGF administration following brain ischemia significantly alleviated neurological deficits and infarct volume. ${ }^{[38,39]}$ For investigating the role of VEGF gene therapy for ischemic stroke, Bellomo et al., ${ }^{[40]}$ injected rAAV-VEGF165 to the lateral ventricle of mice tGCI model six days or 12 days before ischemia and reported that gene therapy significantly improved animal survival, CA1 delayed neuronal death and post-ischemic learning ability. However, potentially deleterious effects of VEGF might compromise its beneficial actions in stroke. It has been reported that early post-ischemic systemic delivery of VEGF increased BBB leakage and induced brain edema, ${ }^{[41]}$ while VEGF antagonization with the high-affinity VEGF-binding protein mFlt(1-3)-IgG improved neurological outcome as well as reduced cerebral edema and infarct size. ${ }^{[42]}$ For reducing VEGF-induced BBB disruption in cerebral ischemia, Shen et al., ${ }^{[19]}$ recently introduced a hypoxiaresponsive element in rAAV2 vector and investigated rAAV2-mediated VEGF expression under a hypoxiaresponsive promoter control in mice cerebral ischemia model and its neuroprotection; five days after gene transfer via lateral ventricle injection, they found that rAAV2-VEGF expressed specifically around the ischemic core and infarct volume was smaller in the gene therapy group compared with control groups and further study revealed that rAAV-mediated VEGF overexpression in ischemic hemisphere can greatly reduce the number of TUNEL-positive cells, cleaved caspase-3-positive cells and fluoro-Jade B-positive neurons. These results suggest that the utilization of hypoxia-responsive element in rAAV vector may be a reasonable and beneficial strategy for stroke gene therapy, which can limit transgene overexpression only in the ischemic area since VEGF overexpression in non-ischemic brain tissue produces side-effect. And the results of the above experiments were consistent with a previous study, which demonstrated that in the ischemic brain VEGF displayed an acute neuroprotective effect, as well as longer latency effects on survival of new neurons and on angiogenesis. ${ }^{[43]}$ The rAAV vector-mediated gene expression in a slow-onset and long-lasting fashion may be very suitable for rAAV-VEGF gene therapy for stroke because delayed and durable VEGF expression beyond acute ischemia stage avoids inducing serious early cerebral swelling and exerts a long function of neuroprotection and vasoformation.

\section{Other genes}

Moreover, it also reported that transduction of rAAV-Interleukin-1, rAAV-neuroglobin and rAAV-AIP (apoptotic protease activating factor-1 interacting protein) could also significantly reduce infarct size and improve functional outcome in focal cerebral ischemia models by different protective mechanisms, which are still not defined. ${ }^{[44-46]}$ However, the therapeutic effects of these genes using rAAV vector on cerebral ischemia are studied only in a few research studies and more experimental evidence is still necessary to validate their efficacy.

\section{Issues of rAAV-based Gene Therapy for Ischemic Stroke}

Results of the above studies have proved that rAAV is a kind of effective and promising vector system for ischemic stroke gene therapy, which can efficiently mediate a series of therapeutic genes' overexpression in targeted ischemic brain areas. However, some questions still need to be considered and answered in future studies. Firstly, the serotype of rAAV vector in almost all the above experiments involved rAAV2 and no study based on other serotype vector has been reported until now in stroke gene therapy investigation. Recently, more and more researches have shown that rAAV8, rAAV1 and rAAV5 have higher transduction efficiency than rAAV2 in the striatum, hippocampus, globus pallidus, substantia nigra. ${ }^{[6,47]}$ Therefore, it is promising that better efficacy may be achieved using rAAV1, rAAV5, rAAV8 as vectors rather than rAAV2. Secondly, other genes, such as hepatocyte growth factor, heat shock protein, fibroblast growth factor and erythropoietin have been proved to beeffective for stroke therapy with adenovirus vector via different protective mechanisms, but whether they have the same or more 
evidence for efficacy with AAV vector remains unknown and needs further probation. Thirdly, to study potential therapeutic agents, most experiments to date have delivered rAAV vector before ischemia and only limited investigations administrating rAAV-Bcl-2 or rAAV-IL-1 after ischemia showed therapeutic efficacy in stroke models. ${ }^{[35,44]}$ However, protein expression in ischemicreperfused tissue is blunted and delayed relative to nonischemic controls, ${ }^{[48]}$ for ischemia typically inhibits transcriptional and translational processes. Thus, ischemia-mediated interference in rAAV-encoded gene expression may also occur and more post-ischemia gene intervention studies may be necessary in the future to investigate ischemia-induced inhibited effect of rAAV vectors. Fourthly, a recent study showed that immune responses to rAAV must be considered in the design of any gene therapy protocol, although the brain is normally immune privileged owing to the BBB. ${ }^{[49]}$ And approximately $80 \%$ of the human population test positive for antibodies to wild type AAV-2 capsid proteins and in $30-70 \%$ antibodies are present that neutralize the AAV-2 capsid. ${ }^{[49]}$ Therefore, if AAV-2 neutralizing antibodies are present, then the efficacy of rAAV2-based gene therapy for stroke might become quite problematic. It has been reported that utilizing rAAV vectors other than serotypes rAAV2 may be one means to circumvent rAAV2-related immune problem. ${ }^{[50]}$ Finally, direct protection of the ischemic penumbra via intracerebral injection of rAAV vector would have to be weighed against the risks of that injection, including infection, hemorrhage and vector-associated immune responses, so a new noninvasive gene delivery approach is also an important target to explore in the future. The BBB disruption after stroke may allow the vector enter the penumbra easily and Han et al., ${ }^{[51]}$ showed that ischemic penumbra could be transfected efficiently by rAAV infusion into the internal carotid artery, which implied that rAAV catheter delivery to occluded cerebral artery segment may be a good transfer approach. Overall, above complex issues are critical for rAAV-based stroke gene therapy and await an answer before the onset of its clinical gene transfer trial in stroke patient.

\section{Perspective}

Gene therapy with rAAV vectors for ischemic stroke is still in its infancy. However, it had made some progress and displayed its efficacy with a satisfactory biological safety profile in preliminary experimental investigations. Although many hurdles will have to be cleared before its introduction into clinical stroke trials, it shows greatly promising perspective for patients in the future. Regarding the acute ischemic stroke, it is our opinion that despite unlikely emerging as a major breakthrough in early phase of stroke, rAAV vectorbased strategies for gene therapy may be important in the chronic rehabilitation phase. Even though it is not yet clear when rAAV-mediated gene therapy for ischemic stroke will become clinically useful, it still appears likely that progress will be made in steps.

\section{References}

1. Green AR, Shuaib A. Therapeutic strategies for the treatment of stroke. Drug Discov Today 2006;11:681-93.

2. Ooboshi H, Ibayashi S, Takada J, Kumai Y, Iida M. Brain ischemia as a potential target of gene therapy. Exp Gerontol 2003;38:183-7.

3. Takeda S, Takahashi M, Mizukami H, Kobayashi E, Takeuchi K, Hakamata Y, \& al. Successful gene transfer using adeno-associated virus vectors into the kidney: Comparison among adeno-associated virus serotype 1-5 vectors in vitro and in vivo. Nephron Exp Nephrol 2004;96:119-26.

4. Grimm D, Kay MA. From virus evolution to vector revolution: Use of naturally occurring serotypes of adeno-associated virus (AAV) as novel vectors for human gene therapy. Curr Gene Ther 2003;3:281-304.

5. Burger C, Gorbatyuk OS, Velardo MJ, Peden CS, Williams P, Zolotukhin $\mathrm{S}$, e al. Recombinant $\mathrm{AAV}$ viral vectors pseudotyped with viral capsids from serotypes 1, 2 and 5 display differential efficiency and cell tropism after delivery to different regions of the central nervous system. Mol Ther 2004;10:302-17.

6. Broekman ML, Comer LA, Hyman BT, Sena-Esteves M. Adeno-associated virus vectors serotyped with AAV8 capsid are more efficient than AAV-1 or -2 serotypes for widespread gene delivery to the neonatal mouse brain. Neuroscience 2006;138:501-10.

7. Summerford C, Samulski RJ. Membrane-associated heparan sulfate proteoglycan is a receptor for adeno-associated virus type 2 virions. J Virol 1998;72:1438-45.

8. Summerford C, Bartlett JS, Samulski RJ. AlphaVbeta5 integrin: A co-receptor for adeno-associated virus type 2 infection. Nat Med 1999;5: 78-82.

9. Qing K, Mah C, Hansen J, Zhou S, Dwarki V, Srivastava A. Human fibroblast growth factor receptor 1 is a co-receptor for infection by adeno-associated virus 2. Nat Med 1999;5:71-7.

10. Bartlett JS, Wilcher R, Samulski RJ. Infectious entry pathway of adenoassociated virus and adenoassociated virus vectors. J Virol 2000;74: 2777-85.

11. Bartlett JS, Samulski RJ, McCown TJ. Selective and rapid uptake of adeno-associated virus type 2 in brain. Hum Gene Ther 1998;9: 1181-6.

12. Duan D, Li Q, Kao AW, Yue Y, Pessin JE, Engelhardt JF. Dynamin is required for recombinant adeno-associated virus type 2 infection. J Virol 1999;73:10371-6.

13. Sanlioglu S, Benson PK, Yang J, Atkinson EM, Reynolds T, Engelhardt JF. Endocytosis and nuclear trafficking of adeno-associated virus type 2 are controlled by rac1 and phosphatidylinositol-3 kinase activation. J Virol 2000;74:9184-96.

14. Evans CH, Gouze E, Gouze JN, Robbins PD, Ghivizzani SC. Gene therapeutic approaches-transfer in vivo. Adv Drug Deliv Rev 2006;58: 243-58.

15. Leker RR, Soldner F, Velasco I, Gavin DK, Androutsellis-Theotokis A, McKay RD. Long-lasting regeneration after ischemia in the cerebral cortex. Stroke 2007;38:153-61.

16. Warnock JN, Merten OW, Al-Rubeai M. Cell culture processes for the production of viral vectors for gene therapy purposes. Cytotechnology 2006;50:141-62.

17. Howell JM. Is there a future for gene therapy? Neuromuscul Disord 1999;9:102-7.

18. Wang Z, Ma HI, Li J, Sun L, Zhang J, Xiao X. Rapid and highly efficient transduction by double-stranded adeno-associated virus vectors in vitro and in vivo. Gene Ther 2003;10:2105-11.

19. Shen F, Su H, Fan Y, Chen Y, Zhu Y, Liu W, đal. Adeno-associated viralvector-mediated hypoxia-inducible vascular endothelial growth factor gene expression attenuates ischemic brain injury after focal cerebral ischemia in mice. Stroke 2006;37:2601-6. 
20. Wu D. Neuroprotection in experimental stroke with targeted neurotrophins. NeuroRx 2005;2:120-8.

21. Henderson CE, Camu W, Mettling C, Gouin A, Poulsen K, Karihaloo M, \& al. Neurotrophins promote motor neuron survival and are present in embryonic limb bud. Nature 1993;363:266-70.

22. Roussa E, Krieglstein K. GDNF promotes neuronal differentiation and dopaminergic development of mouse mesencephalic neurospheres. Neurosci Lett 2004;361:52-5.

23. Mitsumoto H, Ikeda K, Klinkosz B, Cedarbaum JM, Wong V, Lindsay RM. Arrests of motor neuron disease in Wobbler mice cotreated with CNTF and BDNF. Science 1994;265:1107-10.

24. Yan Q, Elliott J, Snider WD. Brain-derived neurotrophic factor rescues spinal motor neurons from axotomy-induced cell death. Nature 1992;360:753-55

25. Tsukahara T, Yonekawa Y, Tanaka K, Ohara O, Wantanabe S, Kimura T, e al. The role of brain-derived neurotrophic factor in transient forebrain ischemia in the rat brain. Neurosurgery 1994;34:323-31.

26. Andsberg G, Kokaia Z, Klein RL, Muzyczka N, Lindvall O, Mandel RJ. Neuropathological and behavioral consequences of adeno-associated viral vector-mediated continuous intrastriatal neurotrophin delivery in a focal ischemia model in rats. Neurobiol Dis 2002;9:187-204.

27. Gustafsson E, Andsberg G, Darsalia V, Mohapel P, Mandel RJ, Kirik D, et al. Anterograde delivery of brain-derived neurotrophic factor to striatum via nigral transduction of recombinant adeno-associated virus increases neuronal death but promotes neurogenic response following stroke. Eur J Neurosci 2003;17:2667-78.

28. Larsson E, Mandel RJ, Klein RL, Muzyczka N, Lindvall O, Kokaia Z. Suppression of insult-induced neurogenesis in adult rat brain by brainderived neurotrophic factor. Exp Neurol 2002;177:1-8.

29. Kobayashi T, Ahlenius H, Thored P, Kobayashi R, Kokaia Z, Lindvall O. Intracerebral infusion of glial cell line-derived neurotrophic factor promotes striatal neurogenesis after stroke in adult rats. Stroke 2006;37:2361-7.

30. Airaksinen MS, Saarma M. The GDNF family:signalling, biological functions and therapeutic value. Nat Rev Neurosci 2002;3:383-94.

31. Arvidsson A, Kokaia Z, Airaksinen MS, Saarma M, Lindvall O. Stroke induces widespread changes of gene expression for glial cell line-derived neurotrophic factor family receptors in the adult rat brain. Neuroscience 2001;106:27-41.

32. Wang Y, Lin SZ, Chiou AL, Williams LR, Hoffer B.J. Glial cell linederived neurotrophic factor protects against ischemia-induced injury in the cerebral cortex. J Neurosci 1997;17:4341-8.

33. Tsai TH, Chen SL, Chiang YH, Lin SZ, Ma HI, Kuo SW, \& al. Recombinant adeno-associated virus vector expressing glial cell linederived neurotrophic factor reduces ischemia-induced damage. Exp Neurol 2000;166:266-75.

34. Arvidsson A, Kirik D, Lundberg C, Mandel RJ, Andsberg G, Kokaia Z, \& al. Elevated GDNF levels following viral vector-mediated gene transfer can increase neuronal death after stroke in rats. Neurobiol Dis 2003;14:542-56.

35. Shimazaki K, Urabe M, Monahan J, Ozawa K, Kawai N. Adenoassociated virus vector-mediated bcl-2 gene transfer into post-ischemic gerbil brain in vivo: Prospects for gene therapy of ischemia-induced neuronal death. Gene Ther 2000;7:1244-9.
36. Okada T, Shimazaki K, Nomoto T, Matsushita T, Mizukami H, Urabe M, et al. Adeno-associated viral vector-mediated gene therapy of ischemiainduced neuronal death. Met Enzymol 2002;346:378-93.

37. Sun Y, Jin K, Clark KR, Peel A, Mao XO, Chang Q, đal. Adeno-associated virus-mediated delivery of BCL-w gene improves outcome after transient focal cerebral ischemia. Gene Ther 2003;10:115-22.

38. Wang YQ, Guo X, Qiu MH, Feng XY, Sun FY. VEGF overexpression enhances striatal neurogenesis in brain of adult rat after a transient middle cerebral artery occlusion. J Neurosci Res 2007;85:740-7.

39. Kaya D, Gürsoy-Ozdemir Y, Yemisci M, Tuncer N, Aktan S, Dalkara T. VEGF protects brain against focal ischemia without increasing bloodbrain permeability when administered intracerebroventricularly. J Cereb Blood Flow Metab 2005;25:1111-8.

40. Bellomo M, Adamo EB, Deodato B, Catania MA, Mannucei C, Marini H, \& al. Enhancement of expression of vascular endothelial growth factor after adeno-associated virus gene transfer is associated with improvement of brain ischemia injury in the gerbil. Pharmacol Res 2003;48:309-17.

41. Zhang ZG, Zhang L, Jiang Q, Zhang R, Davies K, Powers C, đal. VEGF enhances angiogenesis and promotes blood-brain barrier leakage in the ischemic brain. J Clin Invest 2000;106:829-38.

42. van Bruggen N, Thibodeaux H, Palmer JT, Lee WP, Fu L, Cairns B, \& al. VEGF antagonism reduces edema formation and tissue damage after ischemia/reperfusion injury in the mouse brain. J Clin Invest 1999;104:1613-20.

43. Sun Y, Jin K, Xie L, Childs J, Mao XO, Logvinova A, \&al. VEGF-induced neuroprotection, neurogenesis and angiogenesis after focal cerebral ischemia. J Clin Invest 2003;111:1843-51.

44. Tsai TH, Chen SL, Xiao X, Chiang YH, Lin SZ, Kuo SW, \& al. Gene treatment of cerebral stroke by $\mathrm{rAAV}$ vector delivering IL-1ra in a rat model. Neuroreport 2003;14:803-7.

45. Sun Y, Jin K, Peel A, Mao XO, Xie L, Greenberg DA. Neuroglobin protects the brain from experimental stroke in vivo. Proc Natl Acad Sci USA 2003;100:3497-500.

46. Cao G, Xiao M, Sun F, Xiao X, Pei W, Li J, \& al. Cloning of a novel Apaf-1-interacting protein:a potent suppressor of apoptosis and ischemic neuronal cell death. J Neurosci 2004;24:6189-201.

47. Burger C, Nash K, Mandel RJ. Recombinant adeno-associated viral vectors in the nervous system. Hum Gene Ther 2005;16:781-91.

48. Ooboshi H, Ibayashi S, Takada J, Yao H, Kitazono T, Fujishima M. Adenovirus-mediated gene transfer to ischemic brain: Ischemic flow threshold for transgene expression. Stroke 2001;32:1043-7.

49. Zaiss AK, Muruve DA. Immune responses to adeno-associated virus vectors. Curr Gene Ther 2005;5:323-31.

50. Rivière C, Danos O, Douar AM. Long-term expression and repeated administration of AAV type 1,2 and 5 vectors in skeletal muscle of immunocompetent adult mice. Gene Ther 2006;13:1300-8.

51. Han Z, Zhang S, Li H, Ruan X, Xiao X, Tao W, \& al. Expression of recombinant adeno-associated virus in the brain of rats with a focal embolic stroke via carotid artery. Chin Med J (Engl) 2002;115:1170-4.

Accepted on 03-03-2008

Source(s) of support: The National Natural Science Foundation of China (No.30271330), Conflict of Interest: None declared. 